in rabbit reticulocytes; these have been identified by chromatographic properties as completed $\alpha$-chains and free globin. By treatment with detergent the $a$-chains are released from the ribosomes, and are found to be free of $t \mathrm{RNA}$. It therefore appears that they must have been synthesized at an earlier stage, and it is convincingly surmised that these same ribosomes are in fact producing $\beta$-chains. The $\alpha$-chains evidently combine with the $\beta$-chains while these are yet incomplete; the $\alpha \beta$ complexes-that is to say, free globin in its usual dimeric state-are then released, combine with haem and finally associate to form the $\alpha_{2} \beta_{2}$ tetramer. Labelling experiments indicate that the latter processes may proceed by way of intermediates containing less than their full complement of haem.

This last observation is in good accord with findings on the mechanism of the haem-globin reaction in vitro. Winterhalter and Deranleau (Biochemistry, 6, 3136 ; 1967) have found all three haem-deficient haemoglobin species in mixtures of haem with excess globin. The form in which two of the chains carry haem groups is the most prominent, and was isolated chromatographically. The molecule was shown indeed to be tetrameric, but with an increased tendency to dissociate to dimers at low concentrations. By addition of labelled haemin, and separation of the chains, it was established that the $a$-sub-units were the ones that had already contained haem groups.

The concept of a stable species containing only two haems between four chains receives independent support from the work of Heidrich and Schroeder (Biochim. Biophys. Acta, 147, $389 ; 1967)$, who find that when separated haem and globin are recombined, no less than 25 per cent of such a component is formed; it will combine with haemin only when incubated with the cyanide derivative (which is also the one used throughout by Winterhalter and Deranleau).

As it has long been known that globin synthesis is stimulated by added haem, the differential reactivity of the $\alpha$-and $\beta$-chains may lead to an overall regulatory effect, whereby, for example, the synthesis of $\alpha$-chains is regulated by the haem supply; the availability of the $\alpha$-chains then makes possible release of the $\beta$-chains from the ribosome, with the formation of complete haemoglobin, so that effective synchrony is achieved. It is interesting to note, as Baglioni and Campana also point out, that the $H$-chains of immunoglobulins have been shown similarly to be released from the ribosome only after association with completed $L$-chains. This sequence may therefore constitute a general feature of the biosynthesis of sub-unit proteins.

\section{Flagella Morphogenesis}

\section{from our Cell Biology Correspondent}

CuLIA and flagella from protozoan to mammalian cells have the same basic structural pattern. Two central microtubules surrounded by a ring of nine outer paired tubules run the length of the organelle, forming the basic $9+2$ pattern of the axoneme. Where the axoneme enters the cell body, the two central tubules terminate in a basal plate, but the nine outer doublets are continuous with two of the three tubules in the nine triplets of the basal body.

What specifies this very exact structure? Electron microscopy has shown that the basal bodies somehow act as organizers for the axonemes, but that, of course, leaves unanswered the question of the specification of the basal body structure. In several protozoans the pattern of ciliation on the cell surface appears to be controlled by extranuclear genes, and there are several reports-the latest from Smith-Sonneborn and Plaut (J. Cell Sci., 2, 225; 1967)--that basal bodies, like mitochondria and chloroplasts, contain DNA. This immediately raises the possibility that basal bodies are self-replicating organelles in the sense that the DNA could act, no matter how many steps of RNA and protein synthesis intervene, as an organizer for the sub-unit protein of microtubules and perhaps even specify the microtubular protein as well. But it is far from certain that all basal bodies contain DNA. In the current Journal of Cell Biology $(35,323 ; 1967)$. Outka and Kluss suggest that in the protozoan Tetramitus rostratus the basal bodies are not self replicating and that in this species nuclear DNA, not basal body DNA, acts as an organizer for the assembly of flagella.

Tetramitus has, like the more familiar Naegleria gruberi, the very remarkable capacity of existing either as an amoeboid cell or as a flagellate and of transforming from one form to the other. Within two hours, amoeboid Tetramitus devoid of microtubules can change. into a tetraflagellate cell with cytoplasmic microtubules and a "mouth" supported by a microtubular system. This flagellate form is stable; it both feeds and divides. Thus the transformation process provides a useful system for studying flagella morphogenesis.

The amoeboid Tetramitus is devoid of basal bodies and their formation is the first requirement of transformation. The problem, of course, is to recognize the very earliest stage of basal body formation. What Outka and Kluss have seen in the electron microscope is the association of recognizable developing basal bodies with amorphous material, which on morphological grounds they tentatively identify as unassembled microtubular protein. They have also seen striking and apparently transitory projections from the nucleus to the basal body. They reasonably interpret this as meaning that a nuclear gene product somehow seeds the assembly of microtubular protein into the ordered basal body structure and once this nucleation has occurred the flagella can grow, perhaps by self assembly.

Once the basal body has formed, a pocket of the cell balloons out from the cell surface above it, and, at the same time, axonemal microtubules develop from the basal body into the outgrowth. The central pair of the axoneme always appears before the nine outer doublets, which at early stages are randomly arranged before they become sequentially positioned in the outer ring. As the growing axonemal microtubules often terminate in fine filaments, and as similar filaments are scattered throughout the outgrowth, it seems likely that microtubular development involves first the production of filaments by linear aggregation of sub. unit protein and then their assembly into the wall of the microtubule.

In the absence of correlated biochemical or genetic studies, any interpretation of a dynamic cell process from a series of electron micrographs must be only tentative. Nonetheless, the structure and timing of the association between developing basal bodies and nuclear outgrowths clearly suggest that the nucleus may play a very direct part in flagella morphogenesis in some species. 